\title{
Reduced brain activation during imitation and observation of others in children with pervasive developmental disorder: a pilot study
}

\author{
Aiko Kajiume ${ }^{1 *}$, Shiori Aoyama-Setoyama ${ }^{1}$, Yuri Saito-Hori ${ }^{2}$, Nobutsune Ishikawa ${ }^{1}$ and Masao Kobayashi ${ }^{1}$
}

\begin{abstract}
Background: Children with pervasive developmental disorder (PDD) are thought to have poor imitation abilities. Recently, this characteristic has been suggested to reflect impairments in mirror neuron systems (MNS). We used near-infrared spectroscopy (NIRS) to examine the brain activity of children with PDD during tasks involving imitation and observations of others.

Findings: The subjects were 6 male children with PDD (8-14 years old) and 6 age- and gender-matched normal subjects (9-13 years old). A video in which a woman was opening and closing a bottle cap was used as a stimulus. Hemoglobin concentration changes around the posterior part of the inferior frontal gyrus and the adjacent ventral premotor cortex were measured with a 24-channel NIRS machine during action observation and action imitation tasks. Regional oxygenated hemoglobin concentration changes were significantly smaller in the PDD group than in the control group. Moreover, these differences were clearer in the action observation task than in the action imitation task.

Conclusions: Dysfunction in the MNS in children with PDD was suggested by the reduced activation in key MNS regions during tasks involving observations and imitations of others. These preliminary results suggest that further studies are needed to verify MNS dysfunction in children with PDD.
\end{abstract}

Keywords: Pervasive developmental disorder, Imitation, Mirror neuron systems, Near-infrared spectroscopy

\section{Background}

Recent functional brain imaging studies of patients with pervasive developmental disorder (PDD) have indicated that abnormalities in recognition cause various PDD symptoms [1]. In addition, "the broken mirror theory of autism" [2,3] has been proposed after the discovery of mirror neuron systems (MNS). Mirror neurons, which were discovered in monkey F5 and PF areas, are activated when a monkey performs an action and observes it $[4,5]$. These areas are assumed to correspond to the human inferior frontal gyrus (IFG) and inferior parietal lobule [6], respectively, and functional magnetic resonance imaging (fMRI) and electroencephalography (EEG) studies have suggested that these areas contain human mirror neurons $[7,8]$.

\footnotetext{
* Correspondence: aimaru@triton.ocn.ne.jp

'Department of Pediatrics, Hiroshima University Graduate School of Biomedical \& Health Sciences, 1-2-3 Kasumi, Minami-ku, Hiroshima 734-8551, Japan

Full list of author information is available at the end of the article
}

Several studies have used various brain imaging techniques to test this hypothesis e.g., $[9,10]$. However, their results have been controversial. Therefore, further evidence is needed.

Among the many brain imaging techniques, we adopted near-infrared spectroscopy (NIRS) for the following reasons. First, compared to other brain imaging techniques, subjects can move somewhat, and their brain activation is measured in a more natural state. This is important when studying children with PDD because they are hyperkinetic and easily anxious in response to background noise or obstructive spaces.

Therefore, we conducted a NIRS study to explore MNS dysfunction in children with PDD during actionobservation and action-imitation tasks. We enrolled only boys because patients with PDD are predominantly male and data collected from girls might affect the results. We predicted that children with PDD would exhibit 
smaller oxygenated hemoglobin concentration ([oxy-Hb]) changes compared to normal subjects.

\section{Methods}

\section{Subjects}

The subjects were 6 right-handed children with PDD (boys; 8-14 years old; mean, 10.7 years; SD, 2.9 years) and 6 age- and gender-matched normal control subjects (boys; 9-13 years old; mean, 10.9 years; SD, 1.6 years) (Table 1$)$. All children with PDD were outpatients of the Department of Pediatrics, Hiroshima University Hospital, and their conditions were diagnosed according to the criteria of the Diagnostic and Statistical Manual of Mental Disorders, fourth edition. None of the subjects used psychotropic drugs. Normal subjects were recruited through the homepage of the Department of Pediatrics, Hiroshima University. The "social skill test for students [11]," a rating scale often used to assess the social skills of students, was administered to the subjects' parents. This test assesses 4 areas: collective behavior, self-control skills, peer relationships, and communication skills. Ten points in each subscore are equivalent to the age average. The intelligence quotients of the children with PDD were determined with the Wechsler Intelligence Scale for Children, third edition [12]. This study was approved by the Ethical Committee of Clinical Study, Hiroshima University Hospital, and written informed consents were obtained from all subjects' parents before the subjects participated in the study.

\section{Activation tasks}

We made a video in which a woman opens and closes a bottle with a one-touch cap. In the video, she holds the bottle during rest periods and opens and closes the bottle during activation periods. The activation periods were $10 \mathrm{~s}$ long, and they were followed by a 20 -s rest (block design); this was repeated 4 times. Trials began and ended with a 20 -s rest period (Figure 1).

The subjects sat on a chair in front of a 19-inch monitor. In the action-observation task, they only watched the video. In the action-imitation task, the subjects watched the video and imitated her movement. During the rest period, the subjects sat still.

\section{NIRS measurement}

[Oxy-Hb] and deoxygeneted-Hb ([deoxy-Hb]) concentration were measured with a 24-channel NIRS machine (ETG-100; Hitachi Medical Corporation, Tokyo, Japan) at 2 wavelengths of near-infrared light (760 and $840 \mathrm{~nm}$ ). The distance between the pairs of emission and detection probes was $3.0 \mathrm{~cm}$. The NIRS machine can measure $[\mathrm{Hb}]$ changes in a depth of approximately $2-3 \mathrm{~cm}$ (i.e., only the cortical surface area). The changes in [oxy- $\mathrm{Hb}]$ and [deoxy- $\mathrm{Hb}]$ were calculated from the difference in the light absorption characteristics of these chromophores according to the Beer-Lambert law. The absorption of near-infrared light was measured with a time resolution of $0.1 \mathrm{~s}$. Eighteen probes (10 emitters and 8 detectors) were set in 2 probe holders. The holders were placed on the head with the low central probe being positioned at the $\mathrm{T} 3$ or $\mathrm{T} 4$ electrode position of the International 10/20 system used in EEG [13] (Figure 2). The correspondence between the NIRS channels and locations in the cerebral cortex has been previously confirmed by a multi-subject study of anatomical craniocerebral correlation in adults [14], and the T3 and T4 placements corresponded to the bilateral middle temporal gyri with $94-96 \%$ probability.

Table 1 Clinical and demographic details of the subjects

\begin{tabular}{|c|c|c|c|c|c|c|c|}
\hline & \multirow[t]{2}{*}{ Age } & \multirow[t]{2}{*}{ Diagnosis } & \multirow[t]{2}{*}{ IQ } & \multicolumn{4}{|l|}{ Social skill test } \\
\hline & & & & Collective behavior & Self control skills & Peer Relationships & Communication skills \\
\hline \multicolumn{8}{|l|}{$\overline{P D D}$} \\
\hline PDD1 & 8.1 & PDDNOS & 88 & 6 & 7 & 10 & 7 \\
\hline PDD2 & 9.6 & Asperger & 118 & 4 & 5 & 5 & 5 \\
\hline PDD3 & 8.8 & PDDNOS & 115 & 6 & 9 & 8 & 10 \\
\hline PDD4 & 11.2 & Asperger & 110 & 4 & 7 & 3 & 8 \\
\hline PDD5 & 15.3 & PDDNOS & 85 & 2 & 1 & 7 & 3 \\
\hline PDD6 & 10.5 & Asperger & 118 & 5 & 6 & 5 & 8 \\
\hline Mean & 10.7 & & 105.8 & $4.5^{* *}$ & $5.8^{*}$ & $6.3^{* *}$ & $6.8^{*}$ \\
\hline S.D. & 2.9 & & 14.9 & 1.5 & 2.7 & 2.5 & 2.5 \\
\hline \multicolumn{8}{|c|}{ Control subjects $(n=6)$} \\
\hline Mean & 10.9 & & & 10.8 & 10.2 & 12.3 & 11.2 \\
\hline S.D. & 1.6 & & & 2.3 & 1.0 & 1.5 & 2.9 \\
\hline
\end{tabular}

IQ intelligence quotients, PDDNOS Pervasive developmental disorder not otherwise specified. ${ }^{*} p<0.05 ;{ }^{* *} p<0.01$. 


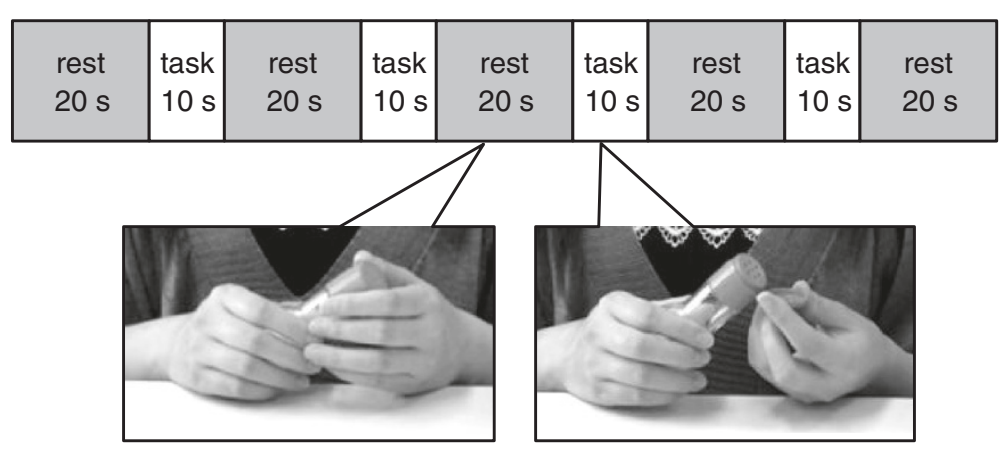

Figure 1 Experimental protocol of the action observation and action imitation tasks.

\section{Statistical analysis}

The $[\mathrm{Oxy}-\mathrm{Hb}]$ data were analyzed because they are considered the most sensitive $[15,16]$. Channels $1,2,11-14,23$, and 24 (i.e., the most anterior and posterior channels of each probe set), in which low signal-to-noise ratios were observed with the exceptional channel function in the NIRS system, were excluded from further analysis.

The data were analyzed with the "integral mode" in the NIRS system to adjust the [oxy-Hb] data for long-term oscillations that were not due to activation tasks. The pretask baseline was determined as the mean value during the 5-s period just before the activation period, and the post-task baseline was determined as the mean value during the last $5 \mathrm{~s}$ of the post-task period. Moving average methods were used to exclude short-term motion artifacts in the analyzed data (moving average window, $5 \mathrm{~s}$ ). The

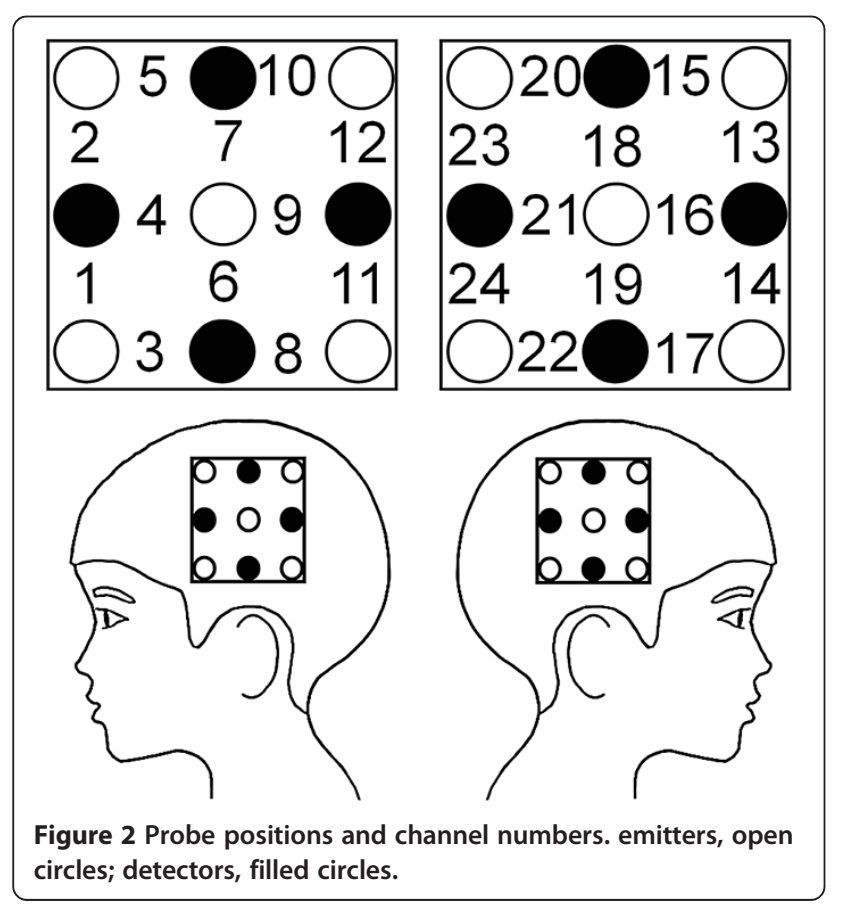

grand average waveforms were calculated with "multiple data analysis" in the NIRS system.

Next, we determined the [oxy-Hb] changes in each channel by calculating the differences in the means from 2.5 to $7.5 \mathrm{~s}$ of the activation period and just before $5 \mathrm{~s}$ of the activation period in order to quantify the neuralinduced concentration changes (hemodynamic response). The [oxy-Hb] changes in each channel were analyzed with a two-way repeated-measures analysis of variance (ANOVA) with "task" (i.e., the action observation and action imitation tasks) as the intra-individual independent variable and "diagnosis" (i.e., PDD and normal control) as the inter-individual independent variable. This ANOVA was exploratory because the subject number was too small to completely justify parametric analysis methods. The level of significance was set at $P<$ 0.05 . For factors that exhibited significance, a post hoc $t$ test was performed; significance levels were corrected by the false-discovery rate method [17].

\section{Results}

All social skill scores were significantly lower in the PDD group compared to the control group (Table 1). Although the PDD group included patients with PDD not otherwise specified and Asperger disorders, these subgroups did not significantly differ for sex, age, social skill scores, and $[\mathrm{oxy}-\mathrm{Hb}]$ changes during the action observation and action imitation tasks.

For the $[\mathrm{oxy}-\mathrm{Hb}]$ changes, the two-way repeatedmeasures ANOVA revealed a significant main effect of "diagnosis" in 5 channels (channels 7, 15, 18, 21, and 22; $F$, 7.3-14.2, $P<0.014$ ) with the significance levels corrected by the false-discovery rate method. The [oxy$\mathrm{Hb}$ ] changes were significantly larger in the control group than in the PDD group, that is, more increasing or less decreasing. The two-way interaction of task by diagnosis did not exhibit statistical significance after correction by the false-discovery rate method, but we found clearer differences between the groups in the actionobservation task than in the action-imitation task 

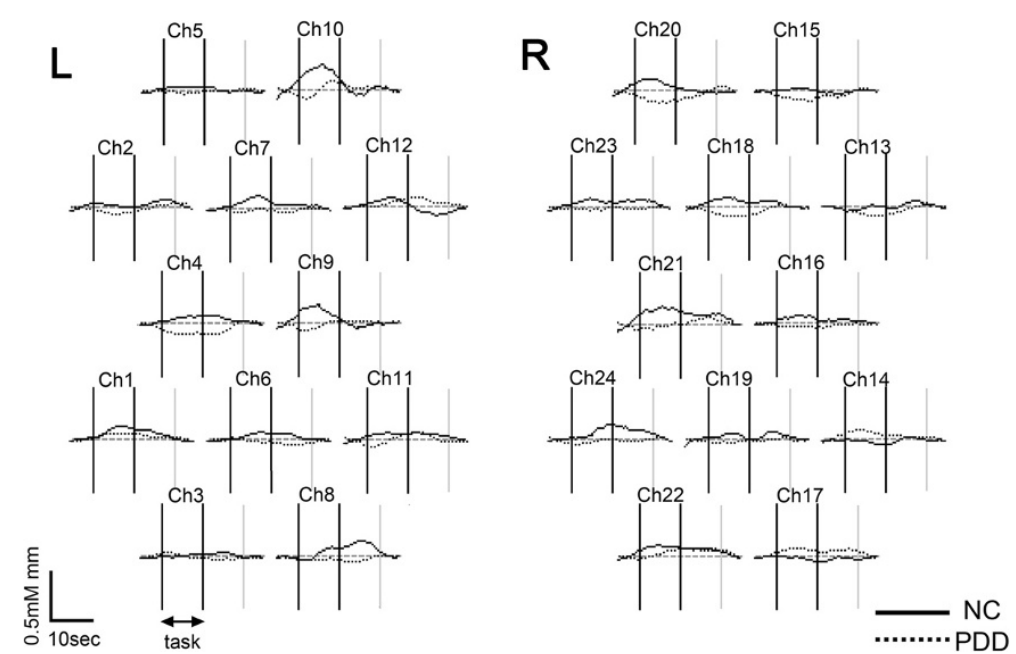

Figure 3 Grand average waveforms of oxygenated hemoglobin concentration changes during the action observation task for controls $(\boldsymbol{N}=6)$ and PDDs $(\boldsymbol{N}=6)$. Controls, solid line; PDD, broken line.

(Figures 3 and 4). The post-hoc $t$-test demonstrated significantly smaller [oxy-Hb] increases or larger decreases in the PDD group than in the control group in 5 channels (channels 7, 15, 18, 21, and 22) during the action observation task, and in 1 channel (channel 15) during the action imitation task in the PDD group than in the control group. Hence, the differences were largely lateralized to the right.

\section{Discussion}

In this preliminary study, we used NIRS to examine the brain function of children with PDD during tasks involving the observation and imitation of others. The results showed that cortical activation during these tasks was significantly smaller in the PDD group than in the control group, and the main differences were on the right hemisphere. We placed the 18 probes so as to cover the posterior part of the IFG and the adjacent ventral premotor cortex (PMC) regions, where human MNS are located [18]. Thus, the channels with significant differences were thought to be relevant to the parts of the MNS. Some studies have shown that mirror neurons function on both sides. Therefore, we placed the probes on both sides of the head. Although the main differences were on the right hemisphere, the channels on the left also showed differences between the groups (e.g., channels 8 and 10). Because this study is preliminary, we need more subjects to study laterality.

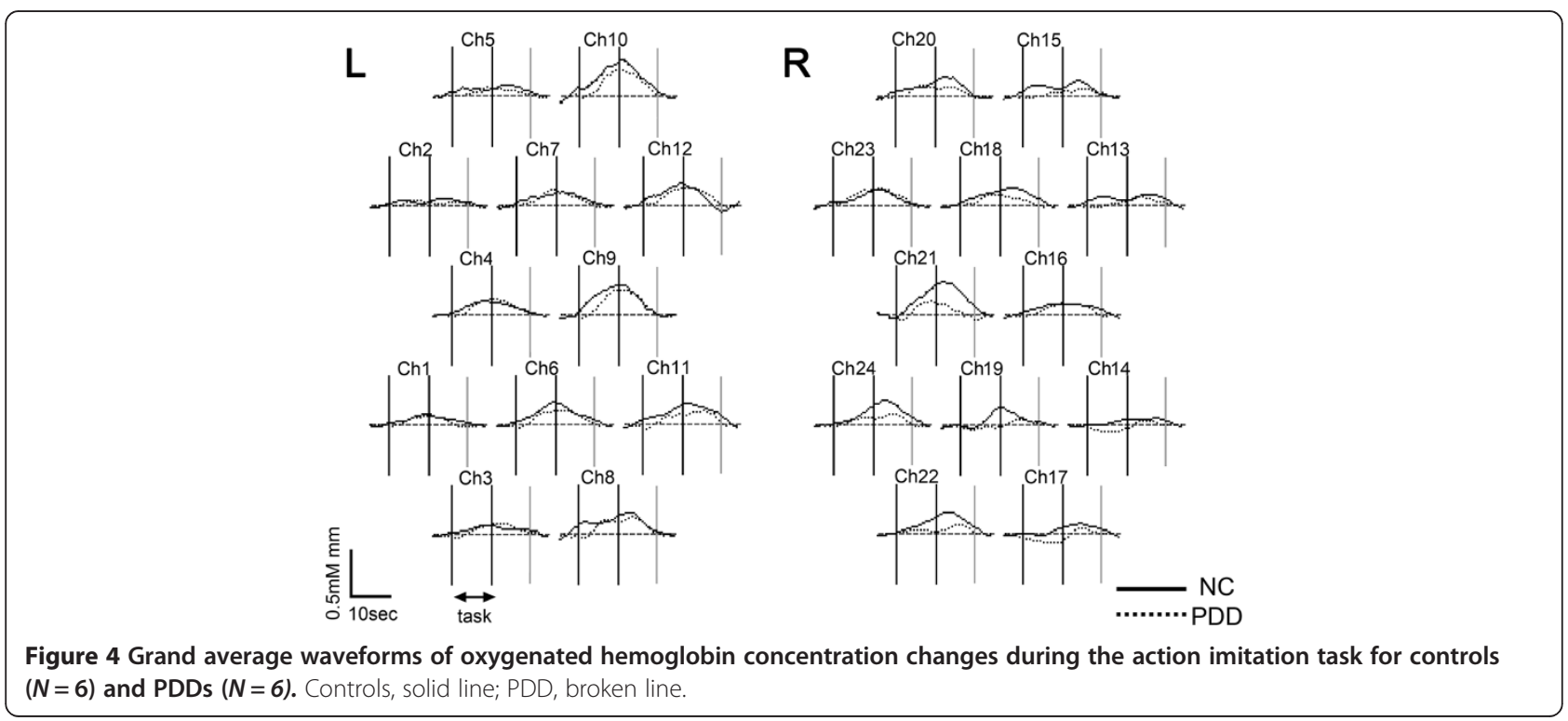


Next, the significant differences between the two groups were clearer in the action-observation task compared to the action-imitation task. This difference may have been influenced by goal-oriented imitation [19], and not automatic imitation, which is impaired in PDD patients. In addition, the consciousness of others, which is weak in PDD children, plays an important role in imitation [20]. With more subjects, we need to explore the differential brain activation between action-observation and imitation tasks.

Our study had several limitations. First, the sample size was small. We should increase the subjects to clarify the validity of this result in the future. Second, the PDD group included subjects with heterogeneous diagnoses. We need to assess differences with respect to diagnoses with more subjects. Third, subject head sizes were smaller than those in past studies of the correspondence between NIRS channels and locations. Other modalities, such as 3-dimensional MRI, could be used in combination with NIRS to precisely analyze the anatomical locations that are activated.

\section{Conclusion}

In this pilot study, brain activation during actionobservation and action-imitation tasks was clearer in the control group than in the PDD group, particularly on the right hemisphere. Further studies are needed to verify the results of this preliminary study and to investigate the mechanisms that underlie the poor imitation abilities of children with PDD.

\section{Ethical approval}

This study was approved by the Ethical Committee of Clinical Study, Hiroshima University Hospital, and written informed consent was obtained from all subjects' parents before their participation in the study.

\section{Competing interests}

All the authors declare that they have no competing interests with respect to this study or its publication.

\section{Author' contributions}

AK designed the study, wrote the protocol, collected the data, statistically analyzed the data, and wrote the first draft of the manuscript. YSH and SAS were involved in working out the study design and data collection, and contributed to writing the final version of the manuscript. NI was involved in analyzing the data and writing the manuscript. MK is the head of our laboratory and contributed to writing the final version of the manuscript. All authors contributed to and have approved the final manuscript.

\section{Acknowledgement}

This research was supported by the Grants-in-Aid for Scientific Research by Japan Society for the Promotion of Science (JSPS). We thank children and their parents for their interested participation. We would like to thank Dr. Junko Tanaka (Hiroshima University Graduate School of Biomedical and Health Sciences) for helpful comments about statistical analysis.

\section{Author details}

${ }^{1}$ Department of Pediatrics, Hiroshima University Graduate School of Biomedical \& Health Sciences, 1-2-3 Kasumi, Minami-ku, Hiroshima 734-8551, Japan. ${ }^{2}$ Department of Child Studies, Sendai Seiyo Gakuin collage, 3-5-75 Itsutsubashi, Wakabayashi-ku, Sendai 984-0022, Japan.
Received: 4 July 2012 Accepted: 20 May 2013

Published: 29 May 2013

\section{References}

1. Baron-Cohen S: The cognitive neuroscience of autism. J Neurol Neurosurg Psychiatr 2004, 75:945-948.

2. Williams $J H$, Whiten A, Suddendorf T, Perrett DI: Imitation, mirror neurons and autism. Neurosci Biobehav Res 2001, 25:287-295.

3. Ramachandran VS, Oberman LM: Broken mirrors: a theory of autism. Sci Am 2006, 295:62-69.

4. Rizzolatti G, Fadiga L, Gallese V, Fogassi L: Premotor cortex and the recognition of motor actions. Cognit Brain Res 1996, 3:131-141.

5. Rizzolatti G, Craighero L: The mirror-neuron system. Annu Rev Neurosci 2004, 27:169-192.

6. Umilta MA, Kohler V, Gallese V, Fogassi L, Fandiga L, Keysers C, Rizzolatti G: I know what you are doing: a neurophysiological study. Neuron 2001, 31:155-165.

7. Hamzei F, Rijntjes M, Dettemers C, Glauche V, Weiller C, Büchel C: The human action recognition system and its relationship to Broca's area: an fMRI study. Neuroimage 2003, 19:637-644.

8. Fecteau S, Carmant L, Tremblay C, Robert M, Bouthiller A, Theoret H: A motor resonance mechanism in children? Evidence from subdural electrodes in a 36-month-old child. Neuroreport 2004, 15:2625-2627.

9. Dapretto M, Davies MS, Pfeifer JH, Scott AA, Sigman M, Bookheimer SY, lacoboni M: Understanding emotions in others: mirror neuron dysfunction in children with autism spectrum disorders. Nat Neurosci 2006, 9:28-30.

10. Hamilton AF, Brindley RM, Frith U: Imitation and action understanding in autism spectrum disorders: how valid is the hypothesis of a deficit in the mirror neuron system? Neuropsychologia 2007, 45:1859-1868.

11. Ueno K, Okada S: The social skill test for student. In The social skill manuals. Tokyo: Meiji-tosyo; 2006:140-143.

12. Japanese WISC-III, Committee P: Japanese Wechsler Intelligence Scale for Children. 3rd edition. Tokyo: Nihon Bunka Kagakusha; 1998.

13. Jasper $\mathrm{HH}$ : The ten-twenty electrode system of the International Federation. Clin Neurophysiol 1958, 10:367-380.

14. Okamoto M, Dan H, Sakamoto K, Takeo K, Shimizu K, Kohno S, Oda I, Isobe S, Suzuki T, Kohyama K, Dan I: Three-dimensional probabilistic anatomical cranio-cerebral correlation via the international 10-20 system oriented for transcranial functional brain mapping. Neuroimage 2004, 21:99-111.

15. Hoshi Y, Kobayashi N, Tamura M: Interpretation of near-infrared spectroscopy signals: a study with a newly developed perfused rat brain model. J Appl Physiol 2001, 90:1657-1662.

16. Saito $Y$, Kondo T, Aoyama S, Fukumoto R, Konishi N, Nakamura K, Kobayashi $\mathrm{M}$, Toshima $\mathrm{T}$ : The function of the frontal lobe in neonates for response to a prosodic voice. Early Hum Dev 2007, 83:225-230.

17. Singh AK, Dan I: Exploring the false discovery rate in multichannel NIRS. Neuroimage 2006, 33:542-549.

18. lacoboni M, Dapretto M: The mirror neuron system and the consequences of its dysfunction. Nat Rev Neurosci 2006, 7:942-951.

19. Hamilton AF: Emulation and mimicry for social interaction: a theoretical approach to imitation in autism. Q J Exp Psychol 2008, 61:101-115.

20. Kana RK, Wadsworth HM, Travers BG: A system level analysis of the mirror neuron hypothesis and imitation impairments in autism spectrum disorders. Neurosci Biobehav Rev 2011, 35:894-902.

doi:10.1186/1744-9081-9-21

Cite this article as: Kajiume et al:: Reduced brain activation during imitation and observation of others in children with pervasive developmental disorder: a pilot study. Behavioral and Brain Functions 2013 9:21. 